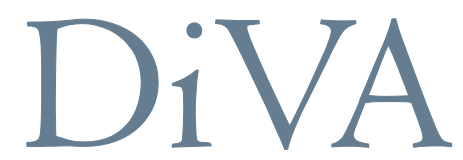

http://uu.diva-portal.org

This is an author produced version of a paper presented at $3^{\text {rd }}$ IFAC Workshop on Distributed Estimation and Control in Networked Systems (Netsys 2012), September 14-15, Santa Barbara, California, USA. This paper has been peer-reviewed but may not include the final publisher proof-corrections or pagination.

Citation for the paper:

Nygren, Johannes, Pelckmans, Kristiaan

"A cooperative decentralized PI control strategy: discrete-time analysis and nonlinear feedback"

In: 3rd IFAC Workshop on Distributed Estimation and Control in Networked Systems, Santa Barbara, CA, 2012 


\title{
A Cooperative Decentralized PI Control Strategy: Discrete-time Analysis and Nonlinear Feedback
}

\author{
Johannes Nygren* Kristiaan Pelckmans * \\ * Division of Systems and Control, Department of Information Technology, \\ Uppsala University, Uppsala, Sweden.
}

\begin{abstract}
This paper discusses an extension of a PI control strategy towards the control of a large $m \times m$-MIMO system. This strategy is fully decentralized, it requires only the tuning of $m$ different controllers, while we only allow for neighboring controllers to exchange error signals. This makes it a strong candidate for an implementation on a decentralized, low-power and high performance Wireless Sensor Network (WSN). The main idea is to feed locally observed control errors ('feedback') not only into the local control law, but also in a fixed proportionate way into neighboring controllers. The analysis concerns convergence to a set point. The analysis is essentially based on a conversion of the PI control law into a discrete-time gradient descent scheme. As an interesting byproduct, this analysis indicates how to deal with quantization functions and nonlinear effects in the feedback signals.
\end{abstract}

The problem of designing and analyzing control strategies using WSNs has been investigated in some depth during the last decade, see e.g. Bemporad et al. (2010) for a survey. For a perspective of automatic control, see e.g. Hespanha et al. (2007). For a discussion of issues of communication and design, see e.g. Fischione et al. (2010). A prominent theoretical theme until date concerns stability of the resulting control law, e.g. Elia and Mitter (2001), while studies of communication issues often involves techniques of quantization (as in Brockett and Liberzon (2000)) and (stochastic) delay (see e.g. Cloosterman et al. (2010)).

In practice, the most prominent way is to use non-cooperative PI(D) controllers implemented on different parts of the MIMO system, see e.g. Palmor et al. (1995). It is indicated that the performance of this approach can be improved considerably by optimization of the loop-pairing of input-output signals which are fed with one univariate control law, see e.g. Åström and Hägglund (2006). This approach is represented in Figure (1). This approach has the advantage of being simple to implement, and to be fully decentralized. However, the rigid structure might not be appropriate for control of strongly interconnected systems.

At the other end of the spectrum, one can find multivariate control laws which control the MIMO system based on techniques of optimal control design or Model Predictive Control (MPC). Such approaches are in general computationally demanding, while they require a centralized node deciding on the control using all information which is gathered from the individual nodes. Despite such strong requirements, this approach is most prevalent in modern distributed control as in Maciejowski (1989).

We are interested in control strategies which strike a good trade-off between the centralized and decentralized extremes. That is, we desire not to be bound by the univariate nature of the decentralized approach, while the application areas in mind (in WSNs) offer neither the computational resources nor the centralized capabilities which are in general required for

\footnotetext{
‡ This work was supported in by the Swedish Foundation for Strategic Research (SSF), project number RIT08-0065.
}

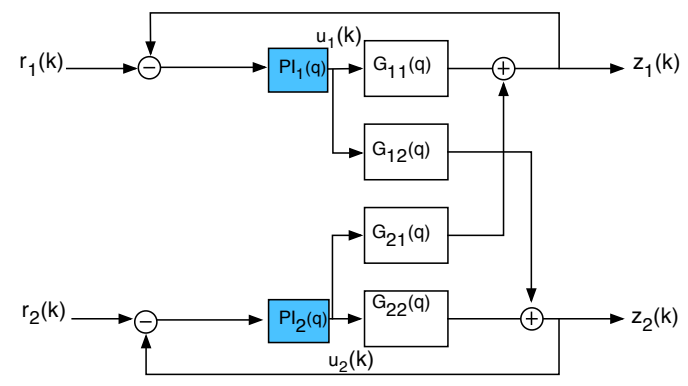

Fig. 1. The traditional design of a decentralized PID control strategy for a $2 \times 2$ MIMO system, with $\left(u_{1}, z_{1}\right)$ and $\left(u_{2}, z_{2}\right)$ paired.

centralized control. The approach adopted here is to take an array of $m$ (P)I control laws and apply them to the $m$ inputoutput signal pairs of the MIMO system at hand. Then we find that a simple modification which allows neighboring controllers to exchange feedback may give us a main boost in performance. This approach is given in Fig. (2). The main contribution of this paper is to provide convergence conditions of the control errors, for a properly chosen diffusion matrix $\mathbf{D}$.

The techniques that we use to analyze such PI rules are based on a reduction of the control law into a gradient descent rule as in Nygren et al. (2012). This is achieved by the construction of a certain matrix $\mathbf{Q}_{m}$. The benefit of doing so is that it gives us access of the wealth of techniques available for gradient descent. Consequently, the result is quite general, and covers many interesting cases, including dealing with Wiener systems, quantization functions and the design of energy-efficient control laws. The other benefit of this technique is that the result concerns the total control error. It is noteworthy that a bound of this performance measure implies (BIBO) stability as well. Finally, we like to point out that this approach makes it possible to compare tuning of PI control laws with techniques of setting the gain in techniques of stochastic approximation, adaptive filtering and optimization.

This paper is organized as follows. The following section details the proposed control strategy, and exemplifies a few 


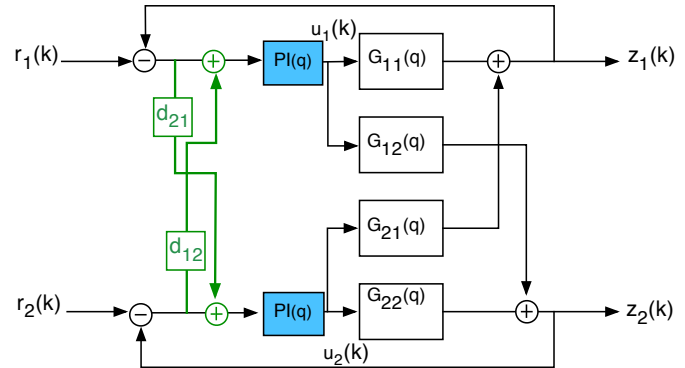

Fig. 2. An example of a collaborative decentralized PI control strategy, where feedback ('errors') are distributed to neighboring controllers as well. That is, errors are rescaled by a factor $d_{i j}$ if going from controller $i$ to neighboring $j$. To keep the figure as simple as possible, we assume here that $d_{11}=d_{22}=1$, and that $f(x)=x$.

interesting cases. Section 3 gives a discrete-time analysis by a reduction to a gradient descent scheme. The technical material on which this result hinges is presented in Appendix A and B. Section 4 gives conclusions and points to further work. The following notational conventions are used. We denote scalars as lowercase letters, vectors are denoted as lower-case boldface letters and matrices are denoted as upper-case, boldface letters. A vector (semi-) norm is denoted as $\|\mathbf{x}\|_{\mathbf{Q}}=\frac{1}{2} \mathbf{x}^{T}\left(\mathbf{Q}^{T}+\mathbf{Q}\right) \mathbf{x}$, for a vector $\mathbf{x}$ and a matrix $\mathbf{Q}$ such that $\mathbf{Q}^{T}+\mathbf{Q}$ is positive (semi)definite (i.e. $\mathbf{Q}^{T}+\mathbf{Q} \succeq 0$ ).

\section{PRELIMINARIES}

\subsection{Setting}

Consider the following linear MIMO system with $m$ inputs and $m$ outputs:

$$
\left[\begin{array}{c}
z_{1}(k) \\
z_{2}(k) \\
\vdots \\
z_{m}(k)
\end{array}\right]=\left[\begin{array}{cccc}
G_{11}(q) & G_{12}(q) & \ldots & G_{1 m}(q) \\
G_{21}(q) & G_{22}(q) & \ldots & G_{2 m}(q) \\
\vdots & \vdots & & \vdots \\
G_{m 1}(q) & G_{m 2}(q) & \ldots & G_{m m}(q)
\end{array}\right]\left[\begin{array}{c}
u_{1}(k) \\
u_{2}(k) \\
\vdots \\
u_{m}(k)
\end{array}\right]
$$

where $u_{i}(k)$ is the $i$ th input (manipulated variable) at time $k \in \mathbb{N}, z_{i}(k)$ is the $i$ 'th output (process variable), and $G_{i j}(q)$ is a discrete time transfer operator describing the influence of the $j$ 'th input on the $i$ 'th output. This paper considers the problem of controlling the $m$ outputs to their respective reference values $\left\{r_{i}\right\}_{i=1}^{m}$ which are assumed to be constant with respect to time.

Let $\mathbf{D} \in \mathbb{R}^{m \times m}$ be a fixed diffusion matrix, such that $\mathbf{D}_{i j}=$ $d_{i j} \in \mathbb{R}$ for all $i, j=1, \ldots, m$. Also let $u_{i}(0), u_{i}(-1), \cdots=0$ for all $i=1, \ldots, m$ (implying that the MIMO system (1) starts from rest). The discrete-time $f \mathbf{D I}$ control law is given as follows.

$$
\begin{gathered}
\varepsilon_{i}(k)=r_{i}-z_{i}(k), \\
u_{i}(k+1)=u_{i}(k)+\gamma \sum_{j=1}^{m} d_{i j} f\left(\varepsilon_{j}(k)\right),
\end{gathered}
$$

where $r_{i}$ is the reference signal (set-point) and $\varepsilon_{i}(k)$ is the control error of the $i$ 'th output signal. The gain at time $k$ is denoted $\gamma$, which is chosen to be larger than zero for all $k$. We see that the control input $i$ depends on a weighting of the control errors $\varepsilon_{j}(k), j=1, \ldots, m$, which is determined by the weighting coefficients $d_{i j}$. The function $f: \mathbb{R} \rightarrow \mathbb{R}$ can, for example, describe quantization of the feedback signal.
Now assume that all subsystems $G_{i j}(q)$ are FIR of finite order $n-1$. Let $\mathbf{h}_{i j}=\left[h_{i j}(0), h_{i j}(1), \ldots, h_{i j}(n)\right]^{T}$ be a $(n+1)$ dimensional column vector containing the Markov parameters $h_{i j}(0), \ldots, h_{i j}(n)$ of $G_{i j}(q)$. Note that $h_{i j}(n)=0$ for all $i$ and $j$ since the order of the subsystems is $n-1$. The reason for including this zero in $\mathbf{h}_{i j}$ will be apparent later.

Let $\mathbf{u}_{k}=\left[u_{1}(k), \ldots u_{1}(k-n), u_{2}(k), \ldots, u_{m}(k-n)\right]^{T}$ be a $m(n+1)$-dimensional column vector where each input from time $k-n$ to $k$ are stacked on each other. Then the $i$ 'th output at time $k$ of (1) can be written concisely as

$$
z_{i}(k)=\left[\mathbf{h}_{i 1}^{T}, \mathbf{h}_{i 2}^{T}, \ldots, \mathbf{h}_{i m}^{T}\right] \mathbf{u}_{k} \equiv \mathbf{h}_{i}^{T} \mathbf{u}_{k} .
$$

Let $\mathbf{r}=\left[r_{1}, r_{2}, \ldots, r_{m}\right]^{T}$ and $\mathbf{z}_{k}=\left[z_{1}(k), z_{2}(k), \ldots, z_{m}(k)\right]^{T}$. Then the control law (2) and (3) can be written concisely as

$$
\begin{gathered}
\varepsilon_{k}=\mathbf{r}-\mathbf{z}_{k}, \\
\mathbf{u}_{k+1}=\mathbf{A}_{m} \mathbf{u}_{k}+\gamma\left\{\mathbf{D} F\left(\varepsilon_{k}\right) \otimes \mathbf{e}_{1}\right\},
\end{gathered}
$$

where $F\left(\varepsilon_{k}\right)=\left[f\left(\varepsilon_{1}(k)\right), f\left(\varepsilon_{2}(k)\right), \ldots, f\left(\varepsilon_{m}(k)\right)\right]^{T}$ and $e_{1}=[1,0, \ldots, 0] \in \mathbb{R}^{m}$. The symbol $\otimes$ is the Kronecker product operator, and the diffusion matrix $\mathbf{D} \in \mathbb{R}^{m \times m}$ equals

$$
\mathbf{D}=\left[d_{i j}\right] \in \mathbb{R}^{m \times m},
$$

where $d_{i j}$ are the weighting coefficients appearing in the $f \mathbf{D I}$ control update (3). Figure 2 shows how a D-matrix (with ones in the diagonal) is represented in a block-diagram of a $2 \times 2$ MIMO system with PI control and linear feedback. Here, $\gamma$ is embedded in the PI-blocks. Note that $\gamma$ acts as a scaling factor on $\mathbf{D}$, and can therefore be embedded in $\mathbf{D}$ as well.

In a decentralized control setting, there are typically structural restrictions on $\mathbf{D}$, depending on how the different controllers can communicate. If the control is completely decoupled, then $\mathbf{D}$ is a diagonal matrix. If any controller can communicate to any other controller, $\mathbf{D}$ is dense.

The matrix $\mathbf{A}_{m} \in \mathbb{R}^{m(n+1) \times m(n+1)}$ is a block-diagonal matrix with $m$ blocks. Such block, denoted as $\mathbf{A} \in \mathbb{R}^{(n+1) \times(n+1)}$, is given as

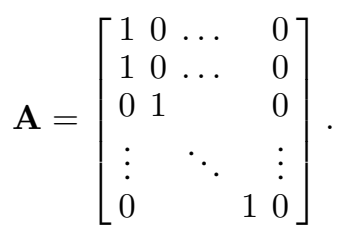

The structure of A is elaborated on in Nygren et al. (2012).

We also assume that the reference signal $\mathbf{r}$ is feasible, i.e. that there exist an input signal $\mathbf{u}^{*}=\left(\boldsymbol{\alpha} \otimes \mathbf{1}_{n+1}\right) \in \mathbb{R}^{m(n+1)}$ such that $\mathbf{h}_{i}^{T} \mathbf{u}^{*}=\sum_{j=1}^{m} \alpha_{j} \mathbf{h}_{i j}^{T} \mathbf{1}_{n+1}=r_{i}$ for all $i$. Note that $\mathbf{h}_{i j}^{T} \mathbf{1}_{n+1}$ is equal to the static gain of subsystem $i j$, i.e. $G_{i j}(1)$. This means that the reference signal is feasible if there exist a solution to the following equation

$$
\left[\begin{array}{ccc}
G_{11}(1) & \ldots & G_{1 m}(1) \\
\vdots & & \vdots \\
G_{m 1}(1) & \ldots & G_{m m}(1)
\end{array}\right] \boldsymbol{\alpha} \equiv \mathbf{G} \boldsymbol{\alpha}=\mathbf{r}
$$

Figure 3 gives a block diagram of the system, the $f$ DI control strategy and the nonlinear feedback loop. The $m \times m$ matrix $\mathbf{G}(q)=\left[G_{i j}(q)\right]$ represents the system transfer operator in (1). This block diagram also motivates the name of the $f \mathrm{DI}$ controller from the ordering of the $f, \mathbf{D}$ and integrator block. This paper will base its analysis on the $f$ DI control strategy, and the analysis requires that the static gain $\mathbf{G}$ is a symmetric matrix. In most applications $\mathbf{G}$ is not symmetric, but Figure 3 


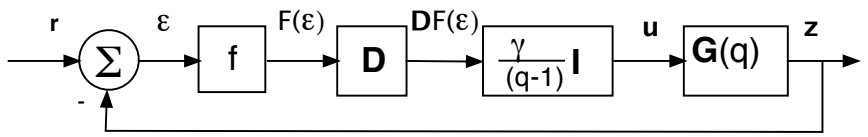

Fig. 3. Block diagram of the MIMO closed loop system. The open loop system is of Hammerstein type, with a (possibly) nonlinear function $f$ followed by a linear part consisting of three blocks.

indicates that one can substitute $\mathbf{G}(q)$ and $\mathbf{D}$ with, for example, $\mathbf{G}^{T} \mathbf{G}(q)$ and $\mathbf{D G}^{-T}$ respectively. The equivalent closed loop system will then have a symmetric static gain.

If one wishes to substitute the DI-control blocks with a more complex controller $\mathbf{C}(q)$, one may factorize it as $\mathbf{C}(q)=$ D $\frac{\gamma}{q-1} \tilde{\mathbf{C}}(q)$ and then merge $\tilde{\mathbf{C}}(q)$ with $\mathbf{G}(q)$. If $\mathbf{C}(q)$ is of PIform, for example, the merged system $\tilde{\mathbf{C}}(q) \mathbf{G}(q)$ will preserve it's FIR form. In the $2 \times 2$ MIMO system in Figure $2, \tilde{\mathbf{C}}(q)$ is a diagonal $2 \times 2$ matrix.

The main questions arising from this discussion are how to tune the parameters of $\tilde{\mathbf{C}}(q)$, how to symmetrize the static gain in the best way (multiplying with $\mathbf{G}^{T}$ is just one possible way), and how to choose the gain $\gamma$ and the diffusion matrix $\mathbf{D}$ subject to some sparsity pattern which represents the possible controller communication pathways. In the forthcoming analysis we restrict ourselves to the problem of choosing $\gamma$ under the assumption that $\mathbf{D}$ and $\mathbf{G}(q)$ are fixed.

\subsection{Special Cases}

The setting given in the previous subsection is general enough to include the following cases of particular interest:

Q: To reduce communication cost in the sensor/actuator network, sensor measurements can be crudely quantized. This can be realized by letting $f(\cdot)$ be a piecewise constant, monotonic stair function:

$$
f(x)=\bar{x}_{i}, \bar{x}_{i} \leq x<\bar{x}_{i+1}, i \in \mathbb{Z} .
$$

In Elia and Mitter (2001), the coarsest quantizer for stabilization is characterized, and it turns out to be of logarithmic form. In this paper, we focus on convergence analysis rather than stabilization.

C: The function $f(\cdot)$ can be any monotone function. For example, if we want to avoid communication at small control errors, it makes sense to choose a dead-zone function with parameter $\delta>0$, defined as

$$
f(x)= \begin{cases}x & |x|>\delta \\ 0 & |x| \leq \delta\end{cases}
$$

$\mathrm{W}$ : It is possible to replace $f\left(\varepsilon_{i}(k)\right)$ with $r_{i}-\tilde{f}\left(z_{i}(k)\right)$, where $\tilde{f}: \mathbb{R} \rightarrow \mathbb{R}$. This is convenient if, for example, the quantized output is communicated between the sensors and actuators instead of the quantized control error. The open loop system is then a Wiener system instead of a Hammerstein system.

\section{ANALYSIS}

Given the setting as described in the previous section, we now want to choose gains $\gamma$ such that the $m$ control errors become as small as possible for a given diffusion matrix $\mathbf{D}$. In this section, we arrive at sufficient conditions on the gain $\gamma$ and function $f$ for asymptotic convergence of the control errors. Now, consider the vector (semi)norm $\|\mathbf{x}\|_{\mathbf{Q}_{m}}^{2}=\frac{1}{2} \mathbf{x}^{T} \mathbf{Q}_{m} \mathbf{x}^{T}$, where $\mathbf{Q}_{m} \in$ $\mathbb{R}^{m(n+1) \times m(n+1)}$. Eq. (6) becomes:

$$
\begin{aligned}
& \left\|\mathbf{u}^{*}-\mathbf{u}_{k+1}\right\|_{\mathbf{Q}_{m}}^{2}=\left\|\mathbf{u}^{*}-\mathbf{u}_{k}\right\|_{\mathbf{A}_{m}^{T} \mathbf{Q}_{m} \mathbf{A}_{m}}^{2} \\
& -2 \gamma\left(\mathbf{u}^{*}-\mathbf{u}_{k}\right)^{T} \mathbf{A}_{m}^{T} \mathbf{Q}_{m}\left\{\mathbf{D} F\left(\varepsilon_{k}\right) \otimes \mathbf{e}_{1}\right\} \\
& +\gamma^{2}\left\|\mathbf{D} F\left(\varepsilon_{k}\right) \otimes \mathbf{e}_{1}\right\|_{\mathbf{Q}_{m}}^{2}
\end{aligned}
$$

Let $\tilde{\mathbf{e}}_{i}=[0, \ldots, 0,1,0, \ldots, 0]^{T} \in \mathbb{R}^{m(n+1)}$, where the 1 is in the $1+(n+1)(i-1)$ entry. As such, the analysis will hinge on the availability of a proper matrix $\mathbf{Q}_{m}$. We will use the following properties of the matrix $\mathbf{Q}_{m}$, and show later the existence of such a matrix.

B1: $\mathbf{Q}_{m}$ is symmetric and positive semidefinite.

B2: for all $i$ we have that $\mathbf{A}_{m}^{T} \mathbf{Q}_{m} \tilde{\mathbf{e}}_{i}=\mathbf{h}_{i}$.

B3: $\mathbf{Q}_{m}-\mathbf{A}^{T} \mathbf{Q}_{m} \mathbf{A}$ is positive semidefinite.

A necessary condition for $\mathbf{Q}_{m}$ to satisfy $\mathbf{B} 2$ is that all $h_{i j}(n)=$ 0 , which we assumed in the Preliminaries section. Appendix A provides a proof that a matrix $\mathbf{Q}_{m}$ which satisfies B1-B3 exist in the SISO case, if and only if the static gain of the system is positive. Together with the feasibility condition (9), we require the static gain of the SISO system to be strictly positive (unless, of course, the reference signal is zero). This positivity constraint is natural since the gain $\gamma$ of the $f$ DI-controller is positive. The case with negative static gain can be dealt with by using negative gains. Appendix B includes an existence proof of $\mathbf{Q}_{m}$ for the MIMO system (1). For existence of $\mathbf{Q}_{m}$ in the MIMO case it is necessary that $\mathbf{G} \succeq 0$. Let $\mathbf{d}_{i}=\left[d_{i 1}, \ldots, d_{i m}\right]^{T}$ equal the $i$ 'th row of $\mathbf{D}$. Using property $\mathrm{B} 2$ and that $\mathbf{r}$ is feasible we make the following derivation of the cross-term in equation (12):

$$
\begin{aligned}
& \left(\mathbf{u}^{*}-\mathbf{u}_{k}\right)^{T} \mathbf{A}_{m}^{T} \mathbf{Q}_{m}\left\{\mathbf{D} F\left(\varepsilon_{k}\right) \otimes \mathbf{e}_{1}\right\}= \\
& \left(\mathbf{u}^{*}-\mathbf{u}_{k}\right)^{T} \mathbf{A}_{m}^{T} \mathbf{Q}_{m}\left\{\tilde{\mathbf{e}}_{1} \otimes \mathbf{d}_{1} F\left(\varepsilon_{k}\right)+\cdots+\tilde{\mathbf{e}}_{m} \otimes \mathbf{d}_{m} F\left(\varepsilon_{k}\right)\right\} \\
& =\varepsilon_{1}(k) \otimes \mathbf{d}_{1} F\left(\varepsilon_{k}\right)+\cdots+\varepsilon_{m}(k) \otimes \mathbf{d}_{m} F\left(\varepsilon_{k}\right) \\
& =\varepsilon_{k}^{T} \mathbf{D} F\left(\varepsilon_{k}\right) .
\end{aligned}
$$

Now let $x_{i j}, i, j=1, \ldots, m$ be the element of $\mathbf{Q}_{m}$ in the $1+(i-1)(n+1)$ 'th row and $1+(j-1)(n+1)$ 'th column (see Appendix B for details). Also let $\mathbf{X}=\left[x_{i j}\right] \in \mathbb{R}^{m \times m}$, and let $\mathbf{x}_{i}$ be the $i$ 'th column of $\mathbf{X}$. Because of $\mathrm{B} 1, \mathbf{X} \succeq 0$ (see Appendix B). Then the last term in (12) becomes

$$
\begin{gathered}
\left\|\mathbf{D} F\left(\varepsilon_{k}\right) \otimes \mathbf{e}_{1}\right\|_{\mathbf{Q}_{m}}^{2} \\
=\left[\sum_{j=1}^{m} \mathbf{d}_{j} F\left(\varepsilon_{k}\right) x_{j 1}, \ldots\right]\left\{\mathbf{D} F\left(\varepsilon_{k}\right) \otimes \mathbf{e}_{1}\right\}^{T} \\
=\left[\sum_{j=1}^{m} \mathbf{d}_{j} F\left(\varepsilon_{k}\right) x_{j 1}, \ldots, \sum_{j=1}^{m} \mathbf{d}_{j} F\left(\varepsilon_{k}\right) x_{j m}\right] \mathbf{D} F\left(\varepsilon_{k}\right) \\
=F\left(\varepsilon_{k}\right)^{T} \mathbf{D}^{T}\left[\mathbf{x}_{1}, \mathbf{x}_{2}, \ldots, \mathbf{x}_{m}\right] \mathbf{D} F\left(\varepsilon_{k}\right) \\
=F\left(\varepsilon_{k}\right)^{T} \mathbf{D}^{T} \mathbf{X D} F\left(\varepsilon_{k}\right) .
\end{gathered}
$$

In the second equality relation in (14) we use the fact that the elements of $\left\{\mathbf{D} F\left(\varepsilon_{k}\right) \otimes \mathbf{e}_{1}\right\}$ between the $1+(i-1)(n+1)$ entries $(i=1, \ldots, m)$ are zero. Using (12) together with (13), (14) and B3, we now arrive at the following expression

$$
\begin{aligned}
& \left\|\mathbf{u}^{*}-\mathbf{u}_{k+1}\right\|_{\mathbf{Q}_{m}}^{2} \leq\left\|\mathbf{u}^{*}-\mathbf{u}_{k}\right\|_{\mathbf{Q}_{m}}^{2} \\
& -2 \gamma \boldsymbol{\varepsilon}_{k}^{T} \mathbf{D} F\left(\varepsilon_{k}\right)+\gamma^{2} F\left(\varepsilon_{k}\right)^{T} \mathbf{D}^{T} \mathbf{X D} F\left(\varepsilon_{k}\right) .
\end{aligned}
$$

This leads to the following lemma, which gives an interesting bound of the control error norms. 
Lemma 1. Given the system (1) where each $G_{i j}(q)$ is FIR of order $\leq n-1$ with static gain $\mathbf{G} \succeq 0$, with the $f \mathbf{D I}$-controller (5) and (6), a feasible reference signal $\mathbf{r}$ and a matrix $\mathbf{Q}_{m}$ which satisfies conditions B1-B3, with an associated $\mathbf{X}$, and assuming that $\mathbf{u}_{0}=\mathbf{0}$, the control errors from time 0 to $k$ will be bounded as

$$
\begin{aligned}
& \sum_{j=0}^{k} \gamma\left(\left\|\varepsilon_{j}\right\|_{\mathbf{D}}^{2}+\left\|F\left(\varepsilon_{j}\right)\right\|_{\mathbf{D}}^{2}-\gamma\left\|F\left(\varepsilon_{j}\right)\right\|_{\mathbf{D}^{T} \mathbf{X D}}^{2}\right) \leq \\
& \boldsymbol{\alpha}^{T} \mathbf{G} \boldsymbol{\alpha}+\sum_{j=0}^{k} \gamma\left\|\varepsilon_{j}-F\left(\varepsilon_{j}\right)\right\|_{\mathbf{D}}^{2}
\end{aligned}
$$

Proof. First we note that $\mathbf{G} \succeq 0$ is equivalent to the existence of a $\mathbf{Q}_{m}$ satisfying B1-B3, according to Appendix B.

Unfolding the recursion (15) gives

$$
\begin{aligned}
& \left\|\mathbf{u}^{*}-\mathbf{u}_{k+1}\right\|_{\mathbf{Q}_{m}}^{2} \leq\left\|\mathbf{u}^{*}-\mathbf{u}_{0}\right\|_{\mathbf{Q}_{m}}^{2} \\
& -2 \sum_{j=0}^{k}\left\{\gamma \boldsymbol{\varepsilon}_{j}^{T} \mathbf{D} F\left(\varepsilon_{j}\right)\right\}+\sum_{j=0}^{k}\left\{\gamma^{2}\left\|F\left(\boldsymbol{\varepsilon}_{j}\right)\right\|_{\mathbf{D}^{T} \mathbf{X D}}^{2}\right\} .
\end{aligned}
$$

Let $\mathbf{q}_{p}$ be the $p$ 'th column of $\mathbf{Q}_{m}$. To develop the first term of (17), we use the fact that $\mathbf{q}_{p}^{T} \mathbf{u}^{*}=\sum_{j=1}^{m} \alpha_{j} \mathbf{1}^{T} \mathbf{h}_{j i}$ for all $p=1+(i-1)(n+1)$ (due to B2) and $\mathbf{q}_{p}^{T} \mathbf{u}^{*}=0$ for all other $p$ (see Appendix A). Since $\mathbf{u}_{0}=\mathbf{0}$, the first term of (17) becomes

$$
\begin{gathered}
\left\|\mathbf{u}^{*}\right\|_{\mathbf{Q}_{m}}^{2}=\left[\sum_{j=1}^{m} \alpha_{j} \mathbf{e}_{1}^{T} \mathbf{h}_{j 1}, 0, \ldots\right] \mathbf{u}^{*} \\
=\sum_{i=1}^{m} \alpha_{i} \sum_{j=1}^{m} \alpha_{j} \mathbf{e}_{1}^{T} \mathbf{h}_{j i}=\boldsymbol{\alpha}^{T} \mathbf{G} \boldsymbol{\alpha} .
\end{gathered}
$$

The cross terms in (17) are replaced by

$$
\begin{aligned}
& 2 \varepsilon_{j}^{T} \mathbf{D} F\left(\varepsilon_{j}\right)= \\
& \left\|\varepsilon_{j}-F\left(\varepsilon_{j}\right)\right\|_{\mathbf{D}}^{2}-\left\|\varepsilon_{j}\right\|_{\mathbf{D}}^{2}-\left\|F\left(\varepsilon_{j}\right)\right\|_{\mathbf{D}}^{2} .
\end{aligned}
$$

Equation (16) is then obtained by using (18) and (19) in (17) and reorganizing the terms.

Lemma 1 states that the control errors will be bounded for all $k=0,1, \ldots$ if $\gamma$ is small enough and if $\left\|\varepsilon_{j}-F\left(\varepsilon_{j}\right)\right\|_{\mathbf{D}}^{2}$ is bounded. The gain $\gamma$ will be small enough to achieve bounded control errors if $F\left(\varepsilon_{j}\right)^{T}\left(\mathbf{D}-\gamma \mathbf{D}^{T} \mathbf{X D}\right) F\left(\varepsilon_{j}\right) \geq 0$ for all $j$, or if $\left(\mathbf{D}^{T}+\mathbf{D}-2 \gamma \mathbf{D}^{T} \mathbf{X D}\right)$ is positive semidefinite. If $F\left(\varepsilon_{k}\right)=$ $\varepsilon_{k}$, we also get asymptotic convergence, i.e. $\lim _{k \rightarrow \infty} \varepsilon_{k}=\mathbf{0}$ if this condition holds.

Now we are ready to state a main result of this paper, which gives conditions for asymptotic convergence of the control errors with respect to $\gamma$ and a proper constraint on the function $f$ (which might represent quantization coarseness as in Elia and Mitter (2001)).

Theorem 2. Given the system (1) where each $G_{i j}(q)$ is FIR of order $\leq n-1$ with static gain $\mathbf{G} \succeq 0$, a matrix $\mathbf{D} \in \mathbb{R}^{m \times m}$ such that $\mathbf{D}^{T}+\mathbf{D} \succeq 0$ and a function $f: \mathbb{R} \rightarrow \mathbb{R}$ that satisfies the following condition

$$
c|\varepsilon| \leq|f(\varepsilon)| \leq|\varepsilon|, \quad \forall \varepsilon \in \mathbb{R}
$$

where $0<c \leq 1$ is chosen such that

$$
\frac{\lambda_{\min }}{\lambda_{\max }}>\frac{(1-c)^{2}}{1+c^{2}},
$$

where $\lambda_{\min }$ and $\lambda_{\max }$ is the minimum and maximum eigenvalue of $\frac{1}{2}\left(\mathbf{D}+\mathbf{D}^{T}\right)$. Then there exist a $\gamma^{*}>0$ such that if the $f$ DI-controller (5) and (6) is applied, with a feasible reference signal $\mathbf{r}$, and assuming that $\mathbf{u}_{0}=\mathbf{0}$, we get asymptotic convergence of the control errors, i.e. $\lim _{k \rightarrow \infty} \varepsilon_{k}=\mathbf{0}$, for all gains satisfying $0<\gamma \leq \gamma^{*}$.

Proof. Without loss of generality, we can assume that $\lambda_{\max }=$ 1 , since $\gamma$ scales with D. By assumption we have that $\lambda_{\text {min }} \geq$ 0 . Also denote the maximum eigenvalue of $\mathbf{D}^{T} \mathbf{X D}$ as $\lambda_{D X D}$.

The main ideas behind the proof is to bound the different quantities of (16) in Lemma 1. Due to (20), we have

$$
\begin{gathered}
\left\|\varepsilon_{k}-F\left(\varepsilon_{k}\right)\right\|_{\mathbf{D}}^{2} \leq\left\|\varepsilon_{k}-F\left(\varepsilon_{k}\right)\right\|^{2} \leq(1-c)^{2}\left\|\varepsilon_{k}\right\|^{2}, \\
\left\|F\left(\varepsilon_{k}\right)\right\|_{\mathbf{D}}^{2} \geq \lambda_{\min }\left\|F\left(\varepsilon_{k}\right)\right\|^{2} \geq \lambda_{\min } c^{2}\left\|\varepsilon_{k}\right\|^{2}
\end{gathered}
$$

and

$$
\left\|F\left(\varepsilon_{k}\right)\right\|_{\mathbf{D}^{T} \mathbf{X D}}^{2} \leq \lambda_{D X D}\left\|F\left(\varepsilon_{k}\right)\right\|^{2} \leq \lambda_{D X D}\left\|\varepsilon_{k}\right\|^{2} .
$$

Then, (16) can be rewritten as

$$
\begin{aligned}
& \left.\gamma\left(\lambda_{\min }\left(1+c^{2}\right)-(1-c)^{2}-\gamma \lambda_{D X D}\right)\right) \sum_{j=0}^{k}\left\|\varepsilon_{j}\right\|^{2} \\
& \leq \boldsymbol{\alpha}^{T} \mathbf{G} \boldsymbol{\alpha} .
\end{aligned}
$$

Due to $(21)$, the quantity $\lambda_{\min }\left(1+c^{2}\right)-(1-c)^{2}$ is strictly positive. Furthermore, since $\mathbf{X} \succeq 0, \lambda_{D X D}$ is nonnegative. Therefore, there exist some number $\gamma^{*}$ such that $0<\gamma \leq \gamma^{*}$ implies that the quantity on the left hand side of (25) is strictly positive. This means that $\sum_{j=0}^{\infty}\left\|\varepsilon_{j}\right\|^{2}<\infty$, implying that $\left\|\varepsilon_{k}\right\|^{2} \rightarrow 0$ as $k \rightarrow \infty$.

\section{DISCUSSION}

This paper proposed a design of cooperative PI control laws for controlling a MIMO system. The key was to have cooperation be encoded in a matrix $\mathbf{D}$, and to work out a reduction to a traditional gradient descent scheme. At this point, this technique is only worked out theoretically for MIMO systems where the components can be represented as FIR systems, while it remains open how to extend to the IIR cases as well.

\section{REFERENCES}

K.J. Åström and T. Hägglund. Advanced PID control. ISA-The Instrumentation, Systems, and Automation Society; Research Triangle Park, NC 27709, 2006.

A. Bemporad, M. Heemels, and M. Johansson. Networked Control Systems, volume 406. Springer-Verlag New York Inc, 2010.

R.W. Brockett and D. Liberzon. Quantized feedback stabilization of linear systems. IEEE Transactions on Automatic Control, 45(7):1279-1289, 2000.

MBG Cloosterman, L. Hetel, N. Van De Wouw, W. Heemels, J. Daafouz, and H. Nijmeijer. Controller synthesis for networked control systems. Automatica, 46(10):1584-1594, 2010.

N. Elia and S.K. Mitter. Stabilization of linear systems with limited information. IEEE Transactions on Automatic Control, 46(9):1384-1400, 2001.

C. Fischione, P. Park, P. Di Marco, and K.H. Johansson. Design principles of wireless sensor networks protocols for control applications. Wireless Networking Based Control, pages 203-238, 2010.

J.P. Hespanha, P. Naghshtabrizi, and Y. Xu. A survey of recent results in networked control systems. Proceedings of the IEEE, 95(1):138-162, 2007.

R.A. Horn and C.R. Johnson. Matrix analysis. Cambridge Univ Pr, 1990.

J.M. Maciejowski. Multivariable feedback design. Electronic Systems Engineering Series, Wokingham, England, Addison-Wesley, 1989.

J. Nygren, K. Pelckmans, and B. Carlsson. A discrete-time PI control law with nonlinear feedback. Submitted, 2012.

Z.J. Palmor, Y. Halevi, and N. Krasney. Automatic tuning of decentralized PID controllers for TITO processes. Automatica, 31(7):1001-1010, 1995. 


\section{Appendix A. EXISTENCE PROOF IN THE SISO CASE}

Here we give an existence proof of a $\mathbf{Q}_{m}$ satisfying B1-B3 in the SISO case. In Appendix B, existence is proven for the MIMO case.

Theorem 3. For a vector $\mathbf{h}=\left[h_{0}, h_{1}, \ldots, h_{n-1}\right]$, there exist a $\mathbf{Q} \in \mathbb{R}^{n+1 \times n+1}$ such that $\mathbf{Q}$ is positive semidefinite (B1), $\mathbf{A}^{T} \mathbf{Q} \mathbf{e}_{1}=\left[\mathbf{h}^{T}, 0\right]^{T}(\mathrm{~B} 2)$ and $\mathbf{Q}-\mathbf{A}^{T} \mathbf{Q A}$ is positive semidefinite (B3), if and only if $\sum_{j=0}^{n-1} h_{j} \geq 0$. $\mathbf{A}$ is defined in (8).

The proof of this theorem is structured in three steps. First, a row sum property of $\mathbf{Q}$ is derived. Then, an equivalent matrix equation is derived using the Jordan form of $\mathbf{A}$. Using the aforementioned row sum property, the condition that $\sum_{j=0}^{n-1} h_{j} \geq 0$ becomes apparent. In the last step of the proof, a specific $\mathbf{Q}_{m}$ which satisfies B1-B3 is derived using the equivalent matrix relation from the second step.

The Q-matrix has the following structure (due to B2):

$$
\mathbf{Q}=\left[\begin{array}{ccccc}
x & h_{0}-x & h_{1} & \ldots & h_{n-1} \\
h_{0}-x & & \tilde{\mathbf{Q}} & & \\
h_{1} & & & \\
\vdots & & & \\
h_{n-1} & & &
\end{array}\right]
$$

where $\tilde{\mathbf{Q}}=\left\{q_{i j}\right\}_{i, j=0}^{n}$ is a symmetric $n \times n$ matrix, and $x$ is a scalar. As a first step, we prove the following proposition.

Proposition 4. Denote $\mathbf{q}_{p}$ as the $p$ 'th row of $\mathbf{Q}$. If $\mathbf{Q}-$ $\mathbf{A}^{T} \mathbf{Q A} \geq 0$ (B3), the sum of all rows of $\mathbf{Q}$ (except for the first row) is zero, i.e. $\mathbf{q}_{p} \mathbf{1}=0$ for all $p=2, \ldots, n+1$. If $\mathbf{A}^{T} \mathbf{Q} \mathrm{e}_{1}=\left[\mathbf{h}^{T}, 0\right]^{T}(\mathrm{~B} 2)$, then $\mathbf{q}_{1} \mathbf{1}=\sum_{j=1}^{n-1} h_{j}$.

Proof. The fact that $\mathbf{q}_{1} \mathbf{1}=\sum_{j=1}^{n-1} h_{j}$ if $\mathrm{B} 2$ holds follows from (A.1). Now observe that $\mathbf{A} \mathbf{1}=\mathbf{1}$. This means that $\mathbf{1}^{T}\left(\mathbf{Q}-\mathbf{A}^{T} \mathbf{Q A}\right) \mathbf{1}=0$ regardless of the properties of $\mathbf{Q}$. By the Rayleigh-Ritz theorem (see Horn and Johnson (1990)), we conclude that if B3 holds, the minimum eigenvalue of $(\mathbf{Q}-$ $\mathbf{A}^{T} \mathbf{Q A}$ ) is zero (with $\mathbf{1}$ as the corresponding eigenvector). This gives the following equations $\mathbf{q}_{1} \mathbf{1}-\mathbf{q}_{1} \mathbf{1}-\mathbf{q}_{2} \mathbf{1}=0$, $\mathbf{q}_{p} \mathbf{1}-\mathbf{q}_{p+1} \mathbf{1}=0$ for all $p=2, \ldots, n$ and $\mathbf{q}_{p+1} \mathbf{1}=0$. These equations imply that $\mathbf{q}_{p} \mathbf{1}=0$ for all $p=2, \ldots, n+1$.

Now we use the following Jordan form transformation on $\mathbf{A}$ :

$$
\mathbf{B}=\mathbf{T}^{-1} \mathbf{A} \mathbf{T},
$$

where

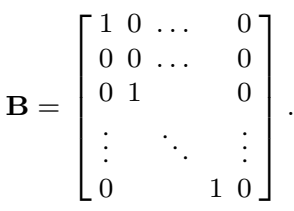

It turns out that

$$
\mathbf{T}=\left[\begin{array}{ccccc}
1 & 0 & \ldots & & 0 \\
1 & -1 & 0 & \ldots & 0 \\
1 & -1 & \ddots & \ddots & \vdots \\
\vdots & \vdots & & & 0 \\
1 & -1 & \ldots & & -1
\end{array}\right]
$$

Now consider the following derivation

$$
\begin{aligned}
& \mathbf{Q}-\mathbf{A}^{T} \mathbf{Q A}=\mathbf{Q}-\mathbf{T}^{-T} \mathbf{B}^{T} \mathbf{T}^{T} \mathbf{Q} \mathbf{T B} \mathbf{T}^{-1} \Leftrightarrow \\
& \mathbf{T}^{T} \mathbf{Q} \mathbf{T}-\mathbf{B}^{T}\left(\mathbf{T}^{T} \mathbf{Q T}\right) \mathbf{B} \equiv \\
& \mathbf{M}-\mathbf{B}^{T} \mathbf{M B} \equiv \mathbf{Z} .
\end{aligned}
$$

Since any matrix $\mathbf{Q}$ and its transformation $\mathbf{T}^{T} \mathbf{Q T}$ has the same inertia (same amount of negative, positive and zero eigenvalues) provided that $\mathbf{T}$ is invertible (which it is), we have that $\mathbf{Q} \succeq 0$ iff $\mathbf{M} \succeq 0$. Furthermore, $\mathbf{Z} \succeq 0$ iff $\mathbf{Q}-\mathbf{A}^{T} \mathbf{Q A} \succeq 0$.

From calculations we get

$$
\mathbf{M}=\left[\begin{array}{cccc}
\sum_{j=0}^{n-1} h_{j} & 0 & \ldots & 0 \\
0 & & \\
\vdots & \tilde{\mathbf{M}}
\end{array}\right]
$$

where the $(k, l)$-entry $\tilde{\mathbf{M}}_{k, l}=\sum_{j=k-1}^{n-1} \sum_{i=l-1} q_{i j}$. The first row and column of $\mathbf{M}$ have zero entries due to Proposition 4. To make $\mathbf{M} \succeq 0$ (and hence $\mathbf{Q} \succeq 0$ ), we see from (A.6) that $\mathrm{B} 1$ holds only if $\sum_{j=0}^{n-1} h_{j} \geq 0$.

Furthermore, we have that

$$
\mathbf{Z}=\left[\begin{array}{cccc}
0 & 0 & \ldots & 0 \\
0 & & \\
\vdots & \tilde{\mathbf{Z}} & \\
0 & &
\end{array}\right]
$$

where $\tilde{\mathbf{Z}}_{k, l}=2 \sum_{j=k-1}^{n-1} q_{j, l-1}-q_{k-1, l-1}$.

The last step of the proof is to give an example of a $\mathbf{Q}$ such that $\mathrm{B} 1$-B3 is satisfied. If we construct a $\mathbf{Q}$ for which $\mathbf{Z} \succeq 0$, we get that $\mathbf{Q}-\mathbf{A}^{T} \mathbf{Q A} \succeq 0$ (B3) and, since the lower principal $n \times n$ submatrix of $\mathbf{B}$ have $n$ zero eigenvalues, $\mathbf{M} \succeq 0$ (which implies B1). Therefore, we construct the following $\overline{\mathbf{Q}}$ :

$$
\mathbf{Q}=\left[\begin{array}{ccccccc}
x & h_{0}-x & h_{1} & h_{2} & & \ldots & h_{n-1} \\
h_{0}-x & x-h_{0}+a_{0} & b_{1}-h_{1} & -h_{2} & & \ldots & -h_{n-1} \\
h_{1} & b_{1}-h_{1} & a_{1} & b_{2} & 0 & \ldots & 0 \\
\vdots & \vdots & & \ddots & & & \\
\vdots & \vdots & & & \ddots & & \\
h_{n-2} & -h_{n-2} & 0 & \ldots & b_{n-2} & a_{n-2} & b_{n-1} \\
h_{n-1} & -h_{n-1} & 0 & \ldots & 0 & b_{n-1} & a_{n-1}
\end{array}\right]
$$

where

$$
\left\{\begin{array}{l}
a_{0}=\sum_{\substack{i=1 \\
n-1}}^{n-1} h_{i}+\sum_{k=1}^{n-1}\left|\sum_{\substack{i=k \\
n-1}}^{n-1} h_{i}\right| \\
a_{j}=\left|\sum_{\substack{i=j \\
n-1}}^{n-1} h_{i}\right|+2 \sum_{k=j+1}\left|\sum_{i=k} h_{i}\right|, \quad j=1, \ldots, n-2 \\
a_{n-1}=\left|h_{n-1}\right|,
\end{array}\right.
$$

and

$$
b_{j}=-\sum_{k=j}^{n-1}\left|\sum_{i=k}^{n-1} h_{i}\right|, \quad j=1, \ldots, n-1 .
$$

We then see that $\tilde{\mathbf{Z}}$ becomes

where 


$$
g(x)=x-\sum_{j=0}^{n-1} h_{j}-\sum_{k=1}^{n-1}\left|\sum_{i=k}^{n-1} h_{i}\right| .
$$

By Gerzgorins disc theorem (see Horn and Johnson (1990)), we see that $\tilde{\mathbf{Z}} \succeq 0$ if $x=\sum_{j=0}^{n-1} h_{j}+2 \sum_{k=1}^{n-1}\left|\sum_{i=k}^{n-1} h_{i}\right|$. Since $\mathbf{Z} \succeq 0$, then $\mathbf{B} 1$ holds (since $\mathbf{M} \succeq 0$ ) and also, $\mathbf{Q}-\mathbf{A}^{T} \mathbf{Q A} \succeq 0$ (B) holds. B2 holds by construction (A.1).

\section{Appendix B. THE MIMO CASE}

In this appendix, an existence proof of a $\mathbf{Q}_{m}$ which satisfies B1$\mathrm{B} 3$ in the MIMO case is given. The formal statement, which is to be proven, is given as the following theorem.

Theorem 5. For given $m(n+1)$-vectors $\mathbf{h}_{i}=\left[\mathbf{h}_{i 1}^{T}, \ldots, \mathbf{h}_{i m}\right]^{T}$, $\mathbf{h}_{i j}=\left[h_{i j}(0), \ldots, h_{i j}(n-1), 0\right]^{T}$ and a $m$-dimensional matrix $\mathbf{G} \equiv\left\{\sum_{k=0}^{n-1} h_{i j}(k)\right\}_{i, j}$, there exist a $\mathbf{Q}_{m} \in \mathbb{R}^{m(n+1) \times m(n+1)}$ such that $\mathbf{Q}_{m}$ is positive semidefinite (B1), $\mathbf{A}_{m}^{T} \mathbf{Q}_{m} \tilde{\mathbf{e}}_{i}=\mathbf{h}_{i}$ for all $i$ (B2) and $\mathbf{Q}_{m}-\mathbf{A}_{m}^{T} \mathbf{Q}_{m} \mathbf{A}_{m}$ is positive semidefinite (B3), if and only if $\mathbf{G} \succeq 0$ (which also means that $\mathbf{G}=\mathbf{G}^{T}$ ).

The structure of the proof is the same as for the SISO case in Appendix A. First, we note that the $m(n+1)$-dimensional matrix $\mathbf{Q}_{m}$ can be divided into $m^{2}$ sub-blocks, which we denote $\mathbf{Q}_{i j} \in \mathbb{R}^{n+1 \times n+1}, i, j=1, \ldots, m$. The sub-block $\mathbf{Q}_{i j}$, which is located at block-row $i$ and block-column $j$ of $\mathbf{Q}_{m}$, has the following structure (due to B2):

$$
\mathbf{Q}_{i j}=\left[\begin{array}{cccc}
x_{i j} & h_{i j}(0)-x_{i j} \ldots & h_{i j}(n-1) \\
h_{j i}(0)-x_{i j} & \tilde{\mathbf{Q}}_{i j} & & \\
h_{j i}(1) & & & \\
\vdots & & & \\
h_{j i}(n-1) & &
\end{array}\right] .
$$

Also note that $\mathbf{Q}_{i j}=\mathbf{Q}_{j i}^{T}$. B1 implies that $\left\{\mathbf{v} \otimes \mathbf{e}_{1}\right\}^{T} \mathbf{Q}_{m}\{\mathbf{v} \otimes$ $\left.\mathbf{e}_{1}\right\}=\mathbf{v}^{T} \mathbf{X} \mathbf{v} \geq 0$ for any vector $\mathbf{v}$, hence $\mathbf{X}$ is positive semidefinite.

As a first step of the proof, we prove the following proposition (which is a generalization of Proposition 4).

Proposition 6. Denote $\mathbf{q}_{i j}^{p}$ as the $p$ 'th row of $\mathbf{Q}_{i j}$. If $\mathbf{Q}_{m}-$ $\mathbf{A}_{m}^{T} \mathbf{Q}_{m} \mathbf{A}_{m} \succeq 0$ (B3), the sum of the rows of $\mathbf{Q}_{i j}, i, j=$ $1, \ldots, m$ (except for the first row) is zero, i.e. $\mathbf{q}_{i j}^{p} \mathbf{1}=0$ for all $p=2, \ldots, n+1$. If $\mathbf{A}_{m}^{T} \mathbf{Q}_{m} \tilde{\mathbf{e}}_{i}=\mathbf{h}_{i}$ (B2), then $\mathbf{q}_{i j}^{1} \mathbf{1}=$ $\sum_{k=1}^{n-1} h_{i j}(k)$.

Proof. The fact that $\mathbf{q}_{i j}^{1} \mathbf{1}=\sum_{k=1}^{n-1} h_{i j}(k)$ if B2 holds follows from (B.1). Now observe that $\mathbf{A}_{m}\left\{\mathbf{e}_{i} \otimes \mathbf{1}_{n+1}\right\}=\left\{\mathbf{e}_{i} \otimes \mathbf{1}_{n+1}\right\}$, where $\mathbf{e}_{i}$ is a $m$-dimensional zero vector except for a 1 at the $i$ 'th element. This means that $\left\{\mathbf{e}_{i} \otimes \mathbf{1}_{n+1}\right\}^{T}\left(\mathbf{Q}-\mathbf{A}^{T} \mathbf{Q A}\right)\left\{\mathbf{e}_{i} \otimes\right.$ $\left.\mathbf{1}_{n+1}\right\}=0$ for all $i=1, \ldots, m$. By the Rayleigh-Ritz theorem, we conclude that if B3 holds, $\left(\mathbf{Q}_{m}-\mathbf{A}_{m}^{T} \mathbf{Q}_{m} \mathbf{A}_{m}\right)\left\{\mathbf{e}_{i} \otimes\right.$ $\left.\mathbf{1}_{n+1}\right\}=\mathbf{0}$ for all $i$. This implies that $\mathbf{q}_{i j}^{1} \mathbf{1}-\mathbf{q}_{i j}^{1} \mathbf{1}-\mathbf{q}_{i j}^{2} \mathbf{1}=0$, $\mathbf{q}_{i j}^{p} \mathbf{1}-\mathbf{q}_{i j}^{p+1} \mathbf{1}=0$ for $p=2, \ldots, n$ and $\mathbf{q}_{i j}^{n+1} \mathbf{1}=0$. These equations imply that $\mathbf{q}_{i j}^{p} \mathbf{1}=0$ for all $p=2, \ldots, n+1$.

Proposition 6 also tells us that if both B2 and B3 holds, then $\sum_{k=0}^{n-1} h_{i j}(k)=\sum_{k=0}^{n-1} h_{j i}(k)$ and hence, $\mathbf{G}$ is symmetric. This is because $\mathbf{Q}_{i j}=\mathbf{Q}_{j i}$ for all $i, j$, implying that the sum of all elements of $\mathbf{Q}_{i j}$ (which is $\sum_{k=0}^{n-1} h_{i j}(k)$ ) is equal to the sum of all elements of $\mathbf{Q}_{j i}$.

In the second step of the proof, we make the the following transformation

$$
\begin{aligned}
& \mathbf{Q}_{m}-\mathbf{A}_{m}^{T} \mathbf{Q}_{m} \mathbf{A}_{m}=\mathbf{Q}_{m}-\mathbf{T}_{m}^{-T} \mathbf{B}_{m}^{T} \mathbf{T}_{m}^{T} \mathbf{Q}_{m} \mathbf{T}_{m} \mathbf{B}_{m} \mathbf{T}_{m}^{-1} \Leftrightarrow \\
& \mathbf{T}_{m}^{T} \mathbf{Q}_{m} \mathbf{T}_{m}-\mathbf{B}_{m}^{T}\left(\mathbf{T}_{m}^{T} \mathbf{Q}_{m} \mathbf{T}_{m}\right) \mathbf{B}_{m} \equiv \\
& \mathbf{M}_{m}-\mathbf{B}_{m}^{T} \mathbf{M}_{m} \mathbf{B}_{m} \equiv \mathbf{Z}_{m} .
\end{aligned}
$$

$\mathbf{B}_{m}$ is the Jordan form of $\mathbf{A}_{m}$, and the transformation matrix $\mathbf{T}_{m}$ is block-diagonal with $m$ blocks of the form (A.4). All sub-blocks $\mathbf{M}_{i j}$ of $\mathbf{M}_{m}$ have the same structure as (A.6). This means that $\left\{\mathbf{v} \otimes \mathbf{e}_{1}\right\}^{T} \mathbf{M}_{m}\left\{\mathbf{v} \otimes \mathbf{e}_{1}\right\}=\mathbf{G}$, and hence, $\mathbf{G} \succeq 0$ is a necessary condition for B1 to hold. Also, all sub-blocks $\mathbf{Z}_{i j}$ of $\mathbf{Z}_{m}$ have the same structure as (A.7).

Now choose $\mathbf{Q}_{m}$ such that

$$
\tilde{\mathbf{Q}}_{i j}=\left[\begin{array}{ccc}
x_{i j}-h_{i j}(0)+\sum_{k=1}^{n-1} h_{j i}(k) & \ldots & -h_{i j}(n-1) \\
-h_{j i}(1) & 0 & \ldots \\
\vdots & & \\
-h_{j i}(n-1) & 0 & \ldots
\end{array}\right]
$$

if $i \neq j$. Also choose $x_{i j}=0$ for $i \neq j$. Then choose $\mathbf{Q}_{i i}$ such that it has the same structure as (A.8), but with the following parameters:

$$
\left\{\begin{array}{l}
a_{0}=\sum_{k=1}^{n-1} h_{i i}(k)+\sum_{j=1}^{m}\left(\sum_{l=1}^{n-1}\left|\sum_{k=l}^{n-1} h_{j i}(k)\right|\right) \\
a_{p}=\sum_{j=1}^{m}\left(\left|\sum_{k=p}^{n-1} h_{j i}(k)\right|+2 \sum_{l=p+1}^{n-1}\left|\sum_{k=l}^{n-1} h_{j i}(k)\right|\right), \\
a_{n-1}=\sum_{j=1}^{m}\left|h_{j i}(n-1)\right|,
\end{array}\right.
$$

and $b_{p}=-\sum_{j=1}^{m} \sum_{l=p}^{n-1}\left|\sum_{k=l}^{n-1} h_{j i}(k)\right|$ for all $j=1, \ldots, n$.

Denote the $i j$-entry of $\mathbf{G}$ as $\mathbf{G}_{i j}$. Then, the sub-blocks $\mathbf{Z}_{i j}$ of $\mathbf{Z}_{m}$ have the same structure as (A.7) with

$$
\tilde{\mathbf{Z}}_{i j}=\left[\begin{array}{ccc}
-\mathbf{G}_{i j} & \ldots & -h_{i j}(n-1) \\
-\sum_{k=1}^{n-1} h_{i j}(k) & 0 & \ldots \\
\vdots & & \\
-h_{n} & 0 & \ldots
\end{array}\right]
$$

for $i \neq j$, and

$$
\tilde{\mathbf{Z}}_{i i}=\left[\begin{array}{cccc}
g_{i i}\left(x_{i i}\right) & -\sum_{k=1}^{n-1} h_{i i}(k) & \ldots & -h_{i i}(n-1) \\
-\sum_{k=1}^{n-1} h_{i i}(k) & \sum_{j=1}^{m}\left|\sum_{k=1}^{n-1} h_{j i}(k)\right| & \ldots \\
\vdots & 0 & \cdots & \sum_{j=1}^{m}\left|h_{j i}(n-1)\right|
\end{array}\right],
$$

where $g_{i i}\left(x_{i i}\right)=x_{i i}-\mathbf{G}_{i i}-\sum_{j=1}^{m}\left(\sum_{l=1}^{n-1}\left|\sum_{k=l}^{n-1} h_{j i}(k)\right|\right)$. By Gerzgorins disc theorem, we see that $Z_{m} \succeq 0$ if

$$
x_{i i}=\sum_{j=1}^{m}\left(\mathbf{G}_{j i}+2 \sum_{l=1}^{n-1}\left|\sum_{k=l}^{n-1} h_{j i}(k)\right|\right) . \square
$$

